\title{
The chemical evolution of self-gravitating primordial disks
}

\author{
Dominik R. G. Schleicher ${ }^{1}$, Stefano Bovino ${ }^{2}$, Muhammad A. Latif ${ }^{3,4}$, Andrea Ferrara ${ }^{5,6}$, and Tommaso Grassi ${ }^{7,8}$ \\ 1 Departamento de Astronomía, Facultad Ciencias Físicas y Matemáticas, Universidad de Concepción, \\ Av. Esteban Iturra s/n Barrio Universitario, Casilla 160-C, Concepción, Chile \\ e-mail: dschleicher@astro-udec.cl \\ 2 Hamburger Sternwarte, University of Hamburg, Gojenbergsweg 112, 21029 Hamburg, Germany \\ 3 Sorbonne Universités, UPMC Univ Paris 06, UMR 7095, Institut d'Astrophysique de Paris, 75014 Paris, France \\ ${ }^{4}$ CNRS, UMR 7095, Institut d'Astrophysique de Paris, 75014 Paris, France \\ 5 Scuola Normale Superiore, Piazza dei Cavalieri 7, 56126 Pisa, Italy \\ 6 Kavli Institute for the Physics and Mathematics of the Universe (WPI), Todai Institutes for Advanced Study, \\ The University of Tokyo, 5-1-5 Kashiwanoha, 277-8583 Kashiwa, Japan \\ 7 Centre for Star and Planet Formation, Natural History Museum of Denmark, Øster Voldgade 5-7, 1350 Copenhagen, Denmark \\ ${ }^{8}$ Niels Bohr Institute, University of Copenhagen, Juliane Maries Vej 30, 2100 Copenhagen, Denmark
}

Received 19 April 2015 / Accepted 7 October 2015

\section{ABSTRACT}

\begin{abstract}
Numerical simulations show the formation of self-gravitating primordial disks during the assembly of the first structures in the Universe, in particular, during the formation of Population III and supermassive stars. Their subsequent evolution is expected to be crucial in determining the mass scale of the first cosmological objects, which depends on the temperature of the gas and dominant cooling mechanism. Here, we derive a one-zone framework to explore the chemical evolution of these disks and show that viscous heating leads to the collisional dissociation of an initially molecular gas. The effect is relevant on scales of $10 \mathrm{AU}$ (1000 AU) for a central mass of $10 M_{\odot}\left(10^{4} M_{\odot}\right)$ at an accretion rate of $10^{-1} M_{\odot} \mathrm{yr}^{-1}$, and provides a substantial heat input to stabilize the disk. If the gas is initially atomic, it remains atomic during the further evolution and the effect of viscous heating is less significant. The additional thermal support is particularly relevant for the formation of very massive objects, such as the progenitors of the first supermassive black holes. The stabilizing impact of viscous heating thus alleviates the need for strong radiation background as a means of keeping the gas atomic.
\end{abstract}

Key words. cosmology: theory - dark ages, reionization, first stars - accretion, accretion disks - astrochemistry - stars: formation stars: Population III

\section{Introduction}

The first stars in the Universe are expected to form from a primordial gas in minihalos of $10^{5}-10^{6} M_{\odot}$ at $z=20-30$ (Abel et al. 2002; Bromm \& Loeb 2003; Yoshida et al. 2008). Their formation process is often explored from cosmological initial conditions, following the gravitational collapse over many orders of magnitude, to scales of astronomical units (AU) or even below. The first stars are expected to be considerably more massive than present day stars owing to the characteristic temperatures of $\sim 300 \mathrm{~K}$ in the primordial gas. Adopting a temperature of $\sim 10 \mathrm{~K}$ in present-day molecular clouds, one may expect an increase in mass by a factor of $30^{1.5} \sim 140$ based on a simple Jeans arguments.

The actual masses and their distribution depend, however, on the details of the star formation process, which are only partly understood. A central question concerns the role of fragmentation, which may lead to the formation of a stellar cluster rather than a central star (Clark et al. 2008). The latter requires the formation of a self-gravitating disk, and its subsequent fragmentation via gravitational instabilities. Indeed, simulations starting from cosmological initial conditions have now confirmed the formation of self-gravitating disks in minihalos as well as their fragmentation (Clark et al. 2011; Greif et al. 2011, 2012; Latif et al. 2013b; Bovino et al. 2014b; Susa et al. 2014). While these simulations followed the evolution for up to $\sim 1000 \mathrm{yr}$ depending on resolution, the Kelvin-Helmholtz timescale for a protostar to reach the main sequence is $\sim 10^{6} \mathrm{yr}$, implying that no final conclusions can be drawn about the properties of the stellar system, and that the simulations so far have only explored the evolution of the disks at very early stages. An exception to this are the studies by Hosokawa et al. (2011) and Hirano et al. (2014), who have followed the formation of primordial stars until they reach the main sequence. These studies accomplished this, however, at the price of a 2D-approximation. This approach provides a hint about the final mass distribution, in particular, if fragmentation is not efficient or if the resulting clumps efficiently merge with the central object. For a further discussion concerning the state of the art of current simulations exploring Population III (Pop. III) star formation, we refer to the recent review by Greif (2015)

In massive primordial halos of $10^{7}-10^{8} M_{\odot}$, it has been suggested that the formation of very massive objects with $\sim 10^{5} M_{\odot}$ occurred (e.g., Bromm \& Loeb 2003; Koushiappas et al. 2004; Lodato \& Natarajan 2006; Begelman \& Shlosman 2009; Natarajan 2011; Volonteri \& Bellovary 2012) as potential progenitors of the observed supermassive black holes at $z \sim 6-7$ (Fan et al. 2001, 2004, 2006; Mortlock et al. 2011; Bañados et al. 2014). In the presence of a strong photodissociation background, particularly high temperatures of the gas can be achieved (e.g., Omukai 2001; Schleicher et al. 2010), therefore, favoring the formation of very massive primordial objects. Simulations 
have shown that self-gravitating disks form in halos cooling via atomic hydrogen (Regan \& Haehnelt 2009; Latif et al. 2013a,c; Prieto et al. 2013; Regan et al. 2014; Becerra et al. 2015), where central masses of $10^{5} M_{\odot}$ can be reached within $10^{4} \mathrm{yr}$ after the initial collapse (Latif et al. 2013a).

The black hole mass function resulting from this kind of a direct collapse has been derived by Ferrara et al. (2014) with a semianalytical approach, considering stellar evolution models for rapidly accreting protostars (Hosokawa et al. 2013; Schleicher et al. 2013). Even for more moderate radiation fluxes, massive stars with $10^{3}-10^{4} M_{\odot}$ may still form (Latif et al. $2014 \mathrm{~b}$ ), and the rotational support in disks was found to increase for an increasing amount of molecular cooling (Latif \& Volonteri 2015). Also, in this context, it is therefore important to understand the long-term evolution of self-gravitating disks both in the atomic and molecular cooling regime.

To complement these numerical investigations, analytical studies have been pursued to investigate the properties of primordial self-gravitating disks. For instance, Lodato \& Natarajan (2006) have discussed the role of self-gravitational instabilities and fragmentation during the formation of a massive central black hole, which may be accompanied with a starburst in the self-gravitating disk. Inayoshi \& Haiman (2014) have explored the fragmentation properties of a self-gravitating disk cooling via atomic hydrogen, concluding that fragments forming via the gravitational instabilities would subsequently merge with the central object. Latif \& Schleicher (2015a) have extended the model into the molecular cooling regime, finding that the merging of clumps is likely still efficient, as the characteristic migration timescales of the clumps are shorter than the timescales of protostellar contraction. In the regime of very massive stars or high accretion rates, the resulting viscous heating may even lead to a transition from a molecular to atomic cooling regime (Latif \& Schleicher 2015b).

Ferrara et al. (2013) have explored the stability of selfgravitating protogalactic disks in the presence of metals, finding that these are subject to strong fragmentation. Self-gravitating protogalactic disks may provide an obstacle for the formation of massive black holes, as suggested by Mayer et al. (2010, 2015). In the context of self-gravitating protostellar disks, the role of metal and dust cooling has been explored by Tanaka \& Omukai (2014), suggesting that they may considerably enhance fragmentation. Similarly, the results of 3D simulations show that fragmentation is triggered by additional cooling, especially for simulations that are evolved beyond the formation of the first peak. This behavior has been observed, for instance, by Clark et al. (2008), Safranek-Shrader et al. (2014), Bovino et al. (2014a), Peters et al. (2014) and Safranek-Shrader et al. (2015).

A substantial debate at this point concerns the question of whether a purely primordial and atomic gas is required for the formation of supermassive black holes, as often assumed in statistical predictions for the high-redshift black hole population (e.g., Dijkstra et al. 2008, 2014; Agarwal et al. 2014). The typical mechanism considered here is the photodissociation of molecular hydrogen by Lyman-Werner radiation, as explored, e.g., by Shang et al. (2010), which is typically parametrized through a critical radiation flux $J_{\text {crit }}$. In recent years, it has been shown that the critical flux can be considerably enhanced through the consideration of realistic stellar spectra (Sugimura et al. 2014; Agarwal et al. 2015) and the dynamics in 3D simulations. In these $3 \mathrm{D}$ simulations, the initial ionization degree is enhanced by shocks and, therefore, requires an even stronger radiation background to prevent the formation of molecular hydrogen (Latif et al. 2014a, 2015a). The simulations suggest a value of $J_{\text {crit }}$ of up to $10^{5}$. For the production of the observed population of $z \sim 6$ black holes, this value is considerably too high, even when considering chemical uncertainties of at most a factor of 5 (Glover 2015).

However, it is by no means clear whether this kind of critical radiation flux is in fact required for the formation of massive objects. For instance, Begelman \& Shlosman (2009) suggested that massive objects could also form from molecular gas in the presence of self-gravitating instabilities. Simulations by Latif et al. (2014b) confirmed that massive objects of $\sim 10^{3}-10^{4} M_{\odot}$ still form for radiation fluxes below $J_{\text {crit }}$, and accretion rates of $\sim 10^{-1} M_{\odot} \mathrm{yr}^{-1}$ can be maintained even for a moderate radiation background (Latif \& Volonteri 2015). In addition, it is conceivable that an atomic gas forms through alternative pathways. For instance, Inayoshi \& Omukai (2012) considered the dissociation of molecular gas by shocks, and showed that the gas would then remain atomic during the further collapse, while Sethi et al. (2010) and Van Borm \& Spaans (2013) considered a similar effect due to the dissipation of magnetic energy.

More recently, we found indications that viscous heating in the presence of rotation can substantially heat up the interior of a self-gravitating disk, leading to a transition toward an atomic cooling regime (Latif \& Schleicher 2015b). The latter can be understood as the viscous heating rate that strongly depends on the angular velocity of the gas, considerably increases toward smaller scales, and ultimately exceeds the cooling rate of a molecular gas. While previous investigations were predominantly exploring a free-fall collapse using one-zone models (e.g., Omukai 2001; Omukai et al. 2005; Glover \& Abel 2008; Glover 2015) or the modeling of the early stages of disk formation (e.g., Regan \& Haehnelt 2009; Clark et al. 2011; Greif et al. 2011, 2012; Latif et al. 2013a,b; Bovino et al. 2014b; Prieto et al. 2013; Regan et al. 2014; Susa et al. 2014; Becerra et al. 2015), we explore the chemical and thermal evolution of these disks at their later stages, in particular, during the presence of a central massive object. For this purpose, we consider different disk models and initial chemical conditions, representing both an initially atomic and molecular gas. To model the chemistry, we employ the publicly available chemistry package KROME $^{1}$ developed by Grassi et al. (2014).

The outline of this paper is as follows. In Sect. 2, we describe the one-zone model for the evolution in a self-gravitating disk in a Lagragian frame as well as the model for the chemical evolution. In Sect. 3, we present our results for the evolution of disks with an initially molecular gas, and Sect. 4 contains the results for initially atomic disks. A summary and discussion are presented in Sect. 5.

\section{Theoretical framework}

In the following, we outline a one-zone model to describe the chemical evolution in the mid-plane of primordial disks. For this purpose, we assume that the disk is axisymmetric and stationary in an Eulerian reference frame. We then consider a ring at radius $R$ with mass $\mathrm{d} M$ and width $\mathrm{d} R$, implying a surface density $\Sigma=\mathrm{d} M /(2 \pi R \mathrm{~d} R)$. In the following, we consider how the gas in the ring moves inward by adopting a Lagrangian frame of reference and we follow its evolution. While the disk is stationary in the Eulerian frame, the gas ring evolves when moving inward, implying that the density, temperature, and chemical abundances is time dependent in the Lagrangian frame.

Webpage KROME: http://kromepackage.org/ 
Depending on the mechanism that provides the effective viscosity of the disk, in the following we describe two models for the evolution of the gas ring. In our first model, we assume that the viscosity is provided by turbulence and/or magnetic fields, leading to a characteristic surface density profile of $\Sigma \propto R^{-1}$ in the stationary case. Our second model, which leads to a steeper density profile, assumes that the viscosity is provided by gravitational stresses.

\subsection{Generic disk model}

As outlined above, we consider a radial annulus of mass $\mathrm{d} M$ and surface density $\Sigma$ at radius $R$ in an axisymmetric primordial disk assumed to be stationary in the Eulerian frame. As a result, the accretion rate $\dot{M}$ is independent of time and position within the disk. The sound speed in the annulus is given as

$c_{\mathrm{s}}=\sqrt{\gamma k_{\mathrm{B}} T / m}$

with $k_{\mathrm{B}}$ the Boltzmann constant, $T$ the gas temperature, and $m$ the mean molecular mass. In the following, we need to construct a dynamical model for the evolution of the annulus, in particular, the radius $R$ and surface density $\Sigma$. The surface density is necessarily approximate, as we are not considering a fully Keplerian disk, but instead a situation in which the disk is also stabilized by turbulent pressure as reflected in the somewhat thick disks forming in primordial environments (see, e.g., Turk et al. 2009; Clark et al. 2011; Greif et al. 2012; Latif et al. 2013b).

In the generic disk model, we initially consider a situation with $\Sigma \propto R^{-1}$, as in the case of a so-called Mestel disk. The latter corresponds to a steady-state solution for a strongly selfgravitating disk with constant sound speed $c_{s}$ and a constant Toomre- $Q$ parameter (Toomre 1964), i.e.,

$Q \sim \frac{c_{\mathrm{s}} \Omega}{\pi G \Sigma}$

on the order of 1 (Bertin 1997; Lodato 2007). Strictly speaking, a Mestel disk further extends to infinity, while we are considering disks of a finite size. The latter also implies that the rotation curve derived from the Mestel disk only holds in an approximate way. While the assumptions employed here are certainly an approximation, for instance, the surface density distribution in realistic disks is often similar to the $R^{-1}$ profile (e.g., Beckwith et al. 1990), and we therefore employ it here in our approximate model. The evolution equation of the disk surface density in an axisymmetric model is now given as (Lodato 2007)

$\frac{\partial \Sigma}{\partial t}=-\frac{1}{R} \frac{\partial}{\partial R}\left[\frac{1}{\left(R^{2} \Omega\right)^{\prime}} \frac{\partial}{\partial R}\left(v \Sigma R^{3} \Omega^{\prime}\right)\right]$,

where the prime' denotes the derivative with respect to $R$. Under the assumption that $v$ and $\Omega$ are independent of $\Sigma$, which is certainly an idealization, it can be shown that the disk surface density evolves on a timescale comparable to the viscous timescale, which is given as

$t_{\mathrm{vis}}=\frac{R^{2}}{v}$

where $v$ the effective viscosity of the gas. Considering the disk annulus in our model, the evolution of the surface density is thus approximately given as

$\dot{\Sigma} \sim \frac{\Sigma}{t_{\mathrm{vis}}}$.
In the case of a Keplerian disk, this expression becomes accurate by including an additional factor of $3 / 2$. We consider more generic situations, however, in which the disk is also stabilized by turbulence and not necessarily dominated by a central source. As we assume here a disk profile with $\Sigma \propto R^{-1}$, the radius of the annulus must follow a similar evolution equation given as

$\dot{R}=-\frac{R}{t_{\text {vis }}}$

For a stationary thin disk, the continuity equation becomes (Lodato 2007)

$\dot{M}=\left|\frac{\mathrm{d} \ln \Omega}{\mathrm{d} \ln R}\right| 2 \pi v \Sigma$.

For a generic disk model, the factor $|\mathrm{d} \ln \Omega / \mathrm{d} \ln R|$ is usually approximiately 1 , and becomes equal to $3 / 2$ for a Keplerian thin disk. We therefore employ the approximate expression

$\dot{M}=3 \pi v \Sigma$.

In our model, we assume that the constant accretion rate $\dot{M}$ is known, and the evolution of the disk surface density follows from Eq. (5). We can therefore employ Eq. (8) to solve for the disk viscosity required to maintain the accretion rate, yielding

$v=\frac{\dot{M}}{3 \pi \Sigma}$.

While this identity follows from the assumption of stationarity and axisymmetry, these conditions can only be achieved if a physical mechanism is present to provide the effective viscosity $v$. The formulation employed here is clearly approximate and the evolution in the disk can depend both on the source of the viscosity, which can be due to turbulence and magnetic fields (see, e.g., Balbus \& Papaloizou 1999; Hawley 2000) or to the presence of gravitational instabilities (Toomre 1964). We assume here that the Toomre $Q$ parameter is at least initially on the order of 1 , implying that self-gravity is relevant when the annulus forms. If the gravitational force is balanced by the centrifugal force, the Keplerian angular velocity in a disk dominated by selfgravity is given as $\Omega_{\mathrm{K}}=\sqrt{2 \pi G \Sigma / R}$ (Lodato 2007). In a realistic disk, however, we expect it to be partly supported by thermal and turbulent pressure, as also demonstrated via numerical simulations (e.g., Latif et al. 2013a,b). For the disk-dominated case, we therefore adopt

$\Omega_{\mathrm{disk}}=\epsilon_{\mathrm{K}} \sqrt{\frac{2 \pi G \Sigma}{R}}$

where $\epsilon_{\mathrm{K}}$ describes the deviation from a fully rotationally supported disk and $G$ denotes the gravitational constant. We assume in the following a generic value $\epsilon_{\mathrm{K}}=50 \%$, as primordial disks typically have scale heights with $H / R$ between $0.3-1 \%$ (Turk et al. 2009; Clark et al. 2011; Greif et al. 2012; Latif et al. $2013 \mathrm{a}, \mathrm{b})$. As we assume that $\Sigma \propto R^{-1}$, the latter implies that the Toomre $Q$ parameter remains $\sim 1$ if the sound speed $c_{\mathrm{s}}$ remains approximately constant. Our surface density profile further implies a non-zero enclosed mass in the innermost region of our disk. For the disk models considered here, the maximum mass within the central $0.1 \mathrm{AU}$ is $0.003 M_{\odot}$, which is not expected to be relevant for the dynamical evolution.

If the disk is dominated by the central source, we similarly assume that the angular velocity is given as a fraction $\epsilon_{\mathrm{K}}$ of the Keplerian rotation, implying that

$\Omega_{\text {source }}=\epsilon_{\mathrm{K}} \sqrt{\frac{G M_{*}}{R^{3}}}$, 
with $M_{*}$ the mass of the central source. In this case, it follows that $Q \propto c_{\mathrm{S}} \Omega / \Sigma \propto R^{-3 / 2} / R^{-1} \propto R^{-1 / 2}$, i.e., $Q$ is increasing with decreasing radius and the interior of the disk becomes gravitationally stable. As long as a sufficient viscosity is provided by turbulence and magnetic fields, the latter is not a problem for the assumed stationarity of the disk. In the next subsection, we consider the possibility that the viscosity is provided by selfgravitational instabilities, which require a steeper relation between $\Sigma$ and $R$. We now calculate the disk height as

$H=\frac{c_{\mathrm{s}}}{\Omega}$.

The latter expression holds for gravitationally stable or marginally unstable disks (Lodato 2007), and is thus valid both for $Q \sim 1$ and $Q>1$. The number density in the mid-plane of the disk follows as

$n=\frac{\Sigma}{2 H m}$,

and the mass density is given as $\rho=n m$. With these quantities, we can also evaluate the viscous heating rate. The latter is given as (Lodato 2007; Ferrara et al. 2013)

$$
Q_{+}=v \Sigma\left(R \Omega^{\prime}\right)^{2} \text {. }
$$

The viscous heating rate therefore generally depends on the rotational profile. In case of rotation around a central source, the viscous heating rate can be evaluated as

$Q_{+, \text {source }}=\frac{9}{4} v \Sigma \Omega_{\text {source }}^{2}$.

For a disk dominated by self-gravity, on the other hand, we obtain $\Omega \propto \sqrt{\Sigma / R} \propto R^{-1}$, implying that

$$
Q_{+, \text {disk }}=v \Sigma \Omega_{\text {disk }}^{2} \text {. }
$$

In this framework, we do not impose thermal equilibrium, and instead follow the nonequilibrum evolution of the gas temperature in our annulus using the detailed chemical model described in Sect. 2.3. The corresponding evolution equation is given as (Grassi et al. 2014)

$$
\frac{\mathrm{d} T}{\mathrm{~d} t}=(\gamma-1) \frac{\Gamma-\Lambda}{k_{\mathrm{B}} n},
$$

where $n$ is the number density of the gas following from disk surface density, disk height and chemical composition, $\Gamma$ is the total heating rate including viscous heating, and $\Lambda$ the total cooling rate. A further description of these expressions is given in Sect. 2.3. While following the nonequilibrium evolution of the system is in principle more accurate than the assumption of thermal equilibrium, the resulting state generally leads to a situation close to thermal equilibrium, i.e., with $\Gamma \sim \Lambda$.

\subsection{Self-regulated disk model}

As already shown above, a disk dominated by a central source is gravitationally stable in the interior if the evolution of the annulus is dictated by Eqs. (5) and (6), implying $\Sigma \propto R^{-1}$. This kind of scenario can thus only be maintained if a sufficient viscosity is provided by turbulence and magnetic fields, as assumed in the generic disk model. However, it is conceivable that gravitational instabilities are required to transport the angular momentum, and they were also shown in previous studies to provide a stronger contribution (e.g., Fromang et al. 2004). While the reality may lie somewhere in between, we explore the extreme case in which self-gravity is necessary to provide the viscosity, requiring that $Q=1$.

In this model, we assume that the disk is gravitationally unstable on scales larger than the current radius $R$, implying that gas is transported onto the annulus from larger scales, and the surface density of the annulus keeps increasing as in Eq. (5). The radius $R$ however remains constant as long as $Q>1$, implying $\dot{R}=0$, and only evolves as in Eq. (6) if $Q \leq 1$. This condition effectively alters the relation between $\Sigma$ and $R$ and increases the surface density until a state of marginal stability is reached. As a result, one obtains a higher surface density and number density on a given scale and the ability of the gas to cool increases compared to the generic disk model outlined above.

\subsection{Chemical model}

To study the chemical evolution for the disk models outlined above, we employ the publicly available chemistry package KROME developed by Grassi et al. (2014). In this framework, we adopt a network similar to that described by Latif et al. (2015a), including the species $\mathrm{H}, \mathrm{H}^{+}, \mathrm{H}^{-}, \mathrm{H}_{2}^{+}, \mathrm{H}_{2}, \mathrm{He}, \mathrm{He}^{+}, \mathrm{He}^{++}$, and $\mathrm{e}^{-}$. We discard photo rates because of the efficiency of self-shielding at high densities. The network includes the currently most accurate rate coefficient for the three-body $\mathrm{H}_{2}$ formation derived by Forrey (2013) from a quantum-mechanical calculation (see also discussion by Bovino et al. 2014c).

The cooling functions in KROME include the atomic line cooling, recombination cooling, and Bremsstrahlung cooling, as described by Cen (1992); the $\mathrm{H}_{2}$ cooling function derived by Glover \& Abel (2008); and the $\mathrm{H}_{2}$ formation heating and cooling, as described by Omukai (2000). Continuum cooling processes are treated, as described by Omukai (2000), with the continuum opacities derived by Lenzuni et al. (1991), and employing a new fit provided by Grassi et al. (2014). The Lenzuni et al. (1991) opacities include all relevant continuum processes, specifically, the bound-free absorption by $\mathrm{H}$ and $\mathrm{H}^{-}$, free-free absorption by $\mathrm{H}, \mathrm{H}^{-}, \mathrm{H}_{2}, \mathrm{H}_{2}^{-}, \mathrm{H}_{2}^{+}, \mathrm{H}_{3}^{+}, \mathrm{He}$, and $\mathrm{He}^{-}$, Rayleigh scattering by $\mathrm{H}, \mathrm{H}_{2}$, and $\mathrm{He}$, and collisionally-induced absorption by $\mathrm{H}_{2}$. The viscous heating is included as described in Sect. 2.

The chemical model described here is invoked within each dynamical timestep of the disk model outlined above to follow the chemical and thermal evolution. As a result, we obtain the evolution of the chemistry while the gas is moving inward. The result is a time sequence in the Lagrangian frame of the annulus, and describes the structure of the stationary disk in the Eulerian frame. In the following, we typically show the results as a function of radius to illustrate the resulting structure of the disk.

\section{Chemical evolution for an initially molecular gas}

In the following, we present the results of the chemical evolution in primordial viscous disks for the scenarios outlined above. For the chemical initial conditions, we consider an initially molecular gas. We adopt a helium fraction of $7.75 \%$ with respect to the total number of nuclei. The typical densities at the beginning of the calculation are $\sim 10^{9}-10^{11} \mathrm{~cm}^{-3}$. For a conservative assessment of the impact of viscous heating, we assume here that the gas is very close to fully molecular, with an atomic hydrogen fraction of only $10^{-6}$. For the electron/proton abundance, we adopt a generic value of $10^{-9}$, which can be expected at these densities (Bovino et al. 2013). The further species abundances, 
D. R. G. Schleicher et al.: The chemical evolution of self-gravitating primordial disks

Table 1. Generic disk models with no central source and an initially molecular gas.

\begin{tabular}{lcccc}
\hline \hline Model & $\dot{M}\left[M_{\odot} \mathrm{yr}^{-1}\right]$ & $\Sigma\left[\mathrm{g} \mathrm{cm}^{-2}\right]$ & $R[\mathrm{AU}]$ & $T[\mathrm{~K}]$ \\
\hline NS0 & $10^{0}$ & 100 & 1000 & 300 \\
NS1 & $10^{-1}$ & 50 & 1000 & 300 \\
NS2 & $10^{-2}$ & 25 & 1000 & 300 \\
NS3 & $10^{-3}$ & 12.5 & 1000 & 300 \\
\hline
\end{tabular}

such as $\mathrm{H}^{-}$and $\mathrm{H}_{2}^{+}$, are initially set to zero, but almost instantaneously reach their equilibrium values during the chemical evolution. We also checked that the results do not strongly depend on these assumptions.

In the following, we present the chemical evolution for such an initially molecular gas for the case of a generic disk model, both within and without a central source and for a self-regulated disk model; we predominantly focus on the case with a central source.

\subsection{Results for a generic disk model with no central source}

For a generic disk model with no central source, we explore a range of scenarios with different accretion rates, varying between $10^{-3}-10^{0} M_{\odot} \mathrm{yr}^{-1}$ and ranging from typical Pop. III star formation (Abel et al. 2002; Bromm \& Loeb 2003) to the conditions in atomic cooling halos (Latif et al. 2013c; Regan et al. 2014). We assume the initial annulus to be at a radius $R=1000 \mathrm{AU}$, with a characteristic temperature of $\sim 300 \mathrm{~K}$. The surface densities of the disk are determined via the model of Latif \& Schleicher (2015b), implying an initial condition with $Q=1$. The details of the models are listed in Table 1 .

As already shown in Sect. 2.1, the surface density scales as $R^{-1}$ under these conditions and the surface densities reach peak values between $10^{5}$ and $10^{6} \mathrm{~g} \mathrm{~cm}^{-2}$ at $R=1 \mathrm{AU}$. The results for this case are given in Fig. 1. We find that the temperature generally increases toward the interior as a result of viscous heating $Q_{+} \propto v \Sigma \Omega_{\text {disk }}^{2}$ (top panel). As $v \propto \Sigma^{-1}$ (Eq. (9)), the heating rate essentially scales as $\Omega_{\text {disk }}^{2} \propto \Sigma / R \propto R^{-2}$, implying a steep increase as a function of radius, which is only partly compensated by the increasing number density of the gas. In the regime considered here, $\mathrm{H}_{2}$ is already thermalized so that the cooling rate only scales linearly with $n_{\mathrm{H}_{2}}$ and optically depth effects increase with density. The gas temperature therefore increases toward values between 1000-2000 K and then rises more gradually.

This behavior is generally found in all cases considered here, with minor deviations for an accretion rate of $0.001 M_{\odot} \mathrm{yr}^{-1}$, where the gas temperature slightly decreases again near $0.1 \mathrm{AU}$ as viscous heating is reduced and collisionally-induced emission (CIE) of molecular hydrogen simultaneously becomes relevant. Furthermore, the temperature of the gas increases with the accretion rate. This is due to the proportional increase in viscous heating, which is only partly compensated by the increased number densities and the ability of the gas to cool. The viscous heating therefore has a relevant impact on the thermal evolution of the gas, even though the differences in the temperature are here smaller than a factor of 2 .

The chemical abundances for a characteristic case with an accretion rate of $0.001 M_{\odot} \mathrm{yr}^{-1}$ (model NS3) are shown in Fig. 1 (middle panel). We find that the gas is fully molecular during the entire evolution. The atomic hydrogen abundance is initially constant, temporarily increases toward $10^{-2}$ around $R \sim 1 \mathrm{AU}$ as a result of the temperature increase, and decreases again in the
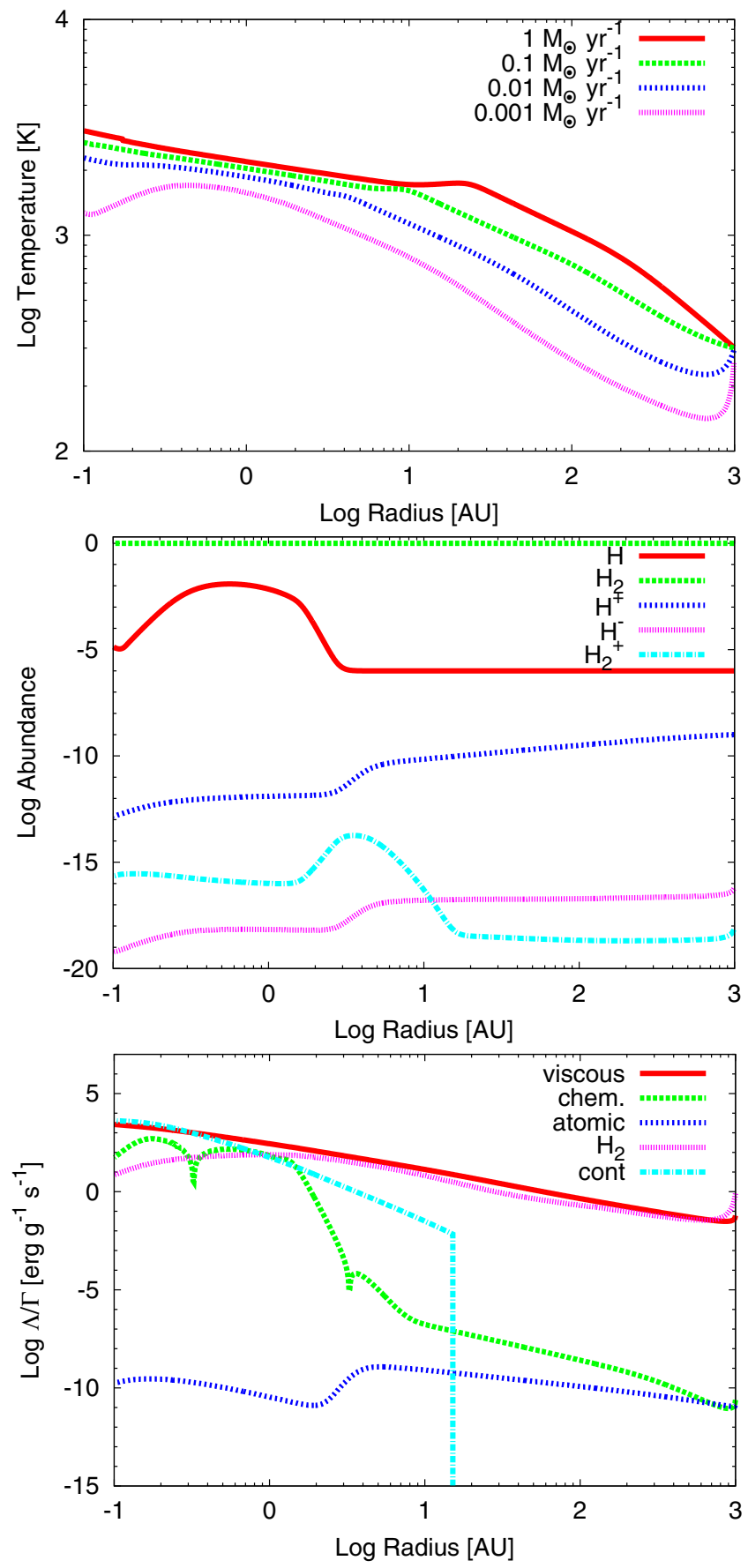

Fig. 1. Results for the generic disk models without central source and an initially molecular gas (Table 1). Top panel: gas temperature vs radius in the case of a generic disk model without central source, assuming different accretion rates. Middle panel: abundances of $\mathrm{H}_{1} \mathrm{H}_{2}, \mathrm{H}^{+}, \mathrm{H}_{2}^{+}$, $\mathrm{H}^{-}$vs. radius for the reference case NS3 (disk with no source, accretion rate $\left.10^{-3} M_{\odot} \mathrm{yr}^{-1}\right)$. Bottom panel: heating and cooling contributions vs radius for the reference case NS3 (disk with no source, accretion rate $10^{-3} M_{\odot} \mathrm{yr}^{-1}$ ). We refer to Table 3 for the heating/cooling contributions defined in the legend.

interior, reflecting the slightly decreasing temperature. Similarly, the proton and electron abundance decreases toward the interior as a result of the increasing densities.

The resulting contributions to heating and cooling are given again for the case of NS3 in Fig. 1 (bottom panel). The viscous heating rate is almost balanced by the molecular hydrogen cooling throughout most of the evolution between 1000 and $1 \mathrm{AU}$, 
Table 2. Models for a generic disk model with central source and an initially molecular gas.

\begin{tabular}{lcccc}
\hline \hline Model & $M_{*}\left[M_{\odot}\right]$ & $\dot{M}\left[M_{\odot} \mathrm{yr}^{-1}\right]$ & $\Sigma\left[\mathrm{g} \mathrm{cm}^{-2}\right]$ & $T[\mathrm{~K}]$ \\
\hline S1A1 & $10^{1}$ & $10^{-1}$ & 10 & 300 \\
S1A3 & $10^{1}$ & $10^{-3}$ & 10 & 300 \\
S2A1 & $10^{2}$ & $10^{-1}$ & 40 & 500 \\
S2A3 & $10^{2}$ & $10^{-3}$ & 40 & 500 \\
S4A0 & $10^{4}$ & $10^{0}$ & 790 & 1900 \\
S4A1 & $10^{4}$ & $10^{-1}$ & 630 & 1100 \\
\hline
\end{tabular}

and subsequently the continuum cooling dominates in the interior. Within $\sim 1 \mathrm{AU}$, we also find strong chemical heating and cooling, which are tightly balanced as the chemistry is close to its equilibrium value. During most of the evolution, the disk remains in the molecular cooling regime without any strong transitions in gas temperature.

\subsection{Results for a generic disk model with central source}

Now we consider the case of a generic disk model with a central source. For this purpose, we consider stellar masses from $10-10^{4} M_{\odot}$, ranging from typical Pop. III to supermassive primordial stars, and accretion rates of $10^{-3}-10^{0} M_{\odot} \mathrm{yr}^{-1}$. We start following the evolution at $R=1000 \mathrm{AU}$, taking the initial surface density and gas temperature from the disk model of Latif \& Schleicher (2015b). The details of the models are listed in Table 2.

In this case, the surface density still scales as $R^{-1}$, reaching peak values of $10^{5}-10^{7} \mathrm{~g} \mathrm{~cm}^{-2}$. The temperature, however, changes more significantly during the evolution. For a characteristic case with a stellar mass of $10 M_{\odot}$ and an accretion rate of $10^{-3} M_{\odot} \mathrm{yr}^{-1}$ (model S1A1), the temperature increases from $300 \mathrm{~K}$ at $R=1000 \mathrm{AU}$ to $\sim 2000 \mathrm{~K}$ at $10 \mathrm{AU}$, and then raises rapidly to $\sim 7000 \mathrm{~K}$ after only a minor change in radius; see Fig. 2 (top panel). This behavior reflects the steep dependence of the viscous heating rate with radius, scaling as $\Omega_{\text {source }}^{2} \propto$ $M_{*} / R^{3}$. The scaling relation is thus considerably steeper than for the case without a central source and increases further with increasing stellar mass. Here, the temperature increases toward a value of $\sim 2000 \mathrm{~K}$, where the collisional dissociation of $\mathrm{H}_{2}$ becomes relevant. The $\mathrm{H}_{2}$ collisional dissociation rate has an exponential dependence on the temperature, and therefore the molecular hydrogen becomes fully dissociated after the temperature increases further. At that point, the gas is no longer able to cool via molecular hydrogen and, therefore, increases until the heating is balanced via atomic cooling. The temperature then remains high at $6000-8000 \mathrm{~K}$ on scales below $10 \mathrm{AU}$.

In general, the evolution is also similar for the other cases considered. The transition toward the atomic cooling regime occurs earlier for higher stellar masses and/or higher accretion rates, as both lead to an increase in the amount of viscous heating. For a $10^{4} M_{\odot}$ star with an accretion rate of $1 M_{\odot} \mathrm{yr}^{-1}$, the viscous heating is particularly efficient, already leading to an almost instantaneous transition at $R \sim 3000 \mathrm{AU}$. This transition toward the atomic cooling regime may considerably increase the stability of the accretion disk in the environment of supermassive stars, as also described by Latif \& Schleicher (2015b).

The chemical abundances for a representative case with a stellar mass of $100 M_{\odot}$ and an accretion rate of $10^{-3} M_{\odot} \mathrm{yr}^{-1}$ (model S2A3) are shown in Fig. 2 (middle panel), clearly showing the transition from the molecular to the atomic regime at $R \sim 30 \mathrm{AU}$. The latter is accompanied by a significant increase in
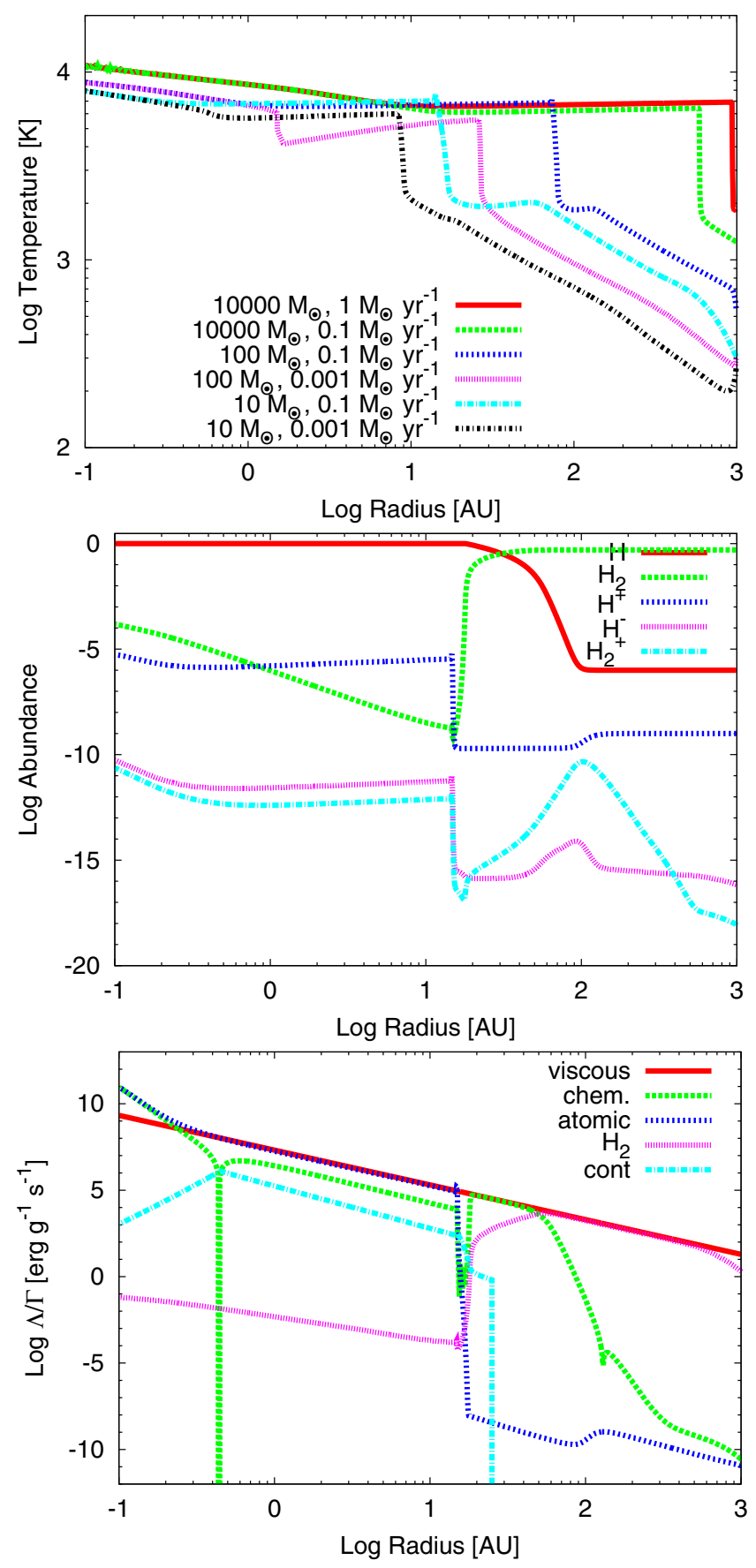

Fig. 2. Results for the generic disk models with central source and an initially molecular gas (Table 2). The species abundances and heating/cooling rates are given for the S2A3 (generic disk model with central source of $100 M_{\odot}$ and accretion rate of $\left.10^{-3} M_{\odot} \mathrm{yr}^{-1}\right)$. We refer to the caption of Fig. 1 for the description of the panels.

the ionization fraction due to the higher temperature. The molecular hydrogen abundance starts increasing again with density after the transition, but does not reach values where the $\mathrm{H}_{2}$ cooling would be significant. In Fig. 2 (bottom panel), the heating and cooling rates are plotted for this reference case, confirming that the cooling is initially dominated by molecular hydrogen and the atomic line cooling takes over after the transition. Within the central $0.3 \mathrm{AU}$, we also find that chemical heating due to $\mathrm{H}_{2}$ formation starts getting significant, but is still balanced by atomic hydrogen cooling. The central temperature then increases to a value of $\sim 9000 \mathrm{~K}$ at $0.1 \mathrm{AU}$. 
D. R. G. Schleicher et al.: The chemical evolution of self-gravitating primordial disks

Table 3. Cooling and heating functions listed under different abbreviations in Figs. 1-5.

\begin{tabular}{l|l|l}
\hline \hline Abbreviation & Cooling and heating terms & Reference \\
\hline Viscous & viscous heating & Lodato (2007) \\
Chem. & net chemical heating/cooling due & Omukai (2000) \\
Chem. heat & chemical heating due to $\mathrm{H}_{2}$ formation & Omukai (2000) \\
Chem. cool & chemical cooling due to $\mathrm{H}_{2}$ dissociation & Omukai (2000) \\
Atomic & atomic line cooling, recombination cooling, Bremsstrahlung & Cen (1992) \\
$\mathrm{H}_{2}$ & $\mathrm{H}_{2}$ line cooling & Glover \& Abel (2008) \\
Cont & continuum cooling & Lenzuni et al. (1991) \\
\hline
\end{tabular}

Table 4. Models for a self-regulated disk with initially molecular gas.

\begin{tabular}{lcccc}
\hline \hline Model & $M_{*}\left[M_{\odot}\right]$ & $\dot{M}\left[M_{\odot} \mathrm{yr}^{-1}\right]$ & $\Sigma\left[\mathrm{g} \mathrm{cm}^{-2}\right]$ & $T[\mathrm{~K}]$ \\
\hline S1 & 1 & $10^{-1}$ & 2.5 & 300 \\
S2 & 10 & $10^{-1}$ & 10 & 400 \\
S3 & $10^{2}$ & $10^{-1}$ & 40 & 500 \\
S4 & $10^{3}$ & $10^{-1}$ & 160 & 800 \\
\hline
\end{tabular}

\subsection{Results for self-regulated disks}

As already discussed in Sect. 2.2, it is conceivable that gravitational instabilities are required to provide a sufficiently large effective viscosity to transport the angular momentum, in particular, in the regime of high accretion rates. We therefore consider the evolution in a self-regulated disk, starting with an initially molecular gas. We predominantly focus on the case with a central source. As we mentioned in Sect. 2.1, the evolution in the case without a central source would be very similar to the generic disk model. We explore characteristic masses of the source of $1-10^{3} M_{\odot}$, including typical Pop. III stars and supermassive stars. Of course, even stronger effects can be expected in the case of more massive stars.

We focus here on typical accretion rates of $10^{-1} M_{\odot} \mathrm{yr}^{-1}$, which are particularly relevant for the formation of supermassive stars in atomic cooling halos and where the heating is particularly strong. We follow the evolution of the disk from a radius of $R=1000 \mathrm{AU}$, and evaluate the initial surface density and gas temperature using the model of Latif \& Schleicher (2015b). As a result of the generally higher surface and number densities in this scenario, we restrict the evolution here to a minimum radius of 1 AU. In fact, considering stellar evolution models for supermassive stars, their typical extent may even exceed this scale (Hosokawa et al. 2013). Overall, a summary of the scenarios considered here is listed in Table 4.

For the self-gravitating disk models, because of the requirement of $Q=1$, the resulting relation between surface density $\Sigma$ and radius $R$ is considerably steeper than for a generic disk model, requiring roughly a relation of $\Sigma \propto R^{-1.5}$. From the results given in Fig. 3 (top panel), it is evident that characteristic features occur when the temperature increases strongly, i.e., at the transition from the molecular to the atomic cooling regime. In this case, the higher gas temperature needs to be compensated by a higher gas surface density to ensure the condition that $Q=1$, thus temporarily steepening the relation between surface density and radius.

For a characteristic case with $1 M_{\odot}$ and an accretion rate of $10^{-1} M_{\odot} \mathrm{yr}^{-1}$ (model S1), the temperature increases first from $400 \mathrm{~K}$ at $1000 \mathrm{AU}$ to $\sim 1800 \mathrm{~K}$ at $30 \mathrm{AU}$. Subsequently, the temperature increases more gradually because of the collisional dissociation cooling of molecular hydrogen toward temperatures above $2000 \mathrm{~K}$ near $3 \mathrm{AU}$, where $\mathrm{H}_{2}$ becomes fully dissociated and the temperature increases to $\sim 5000 \mathrm{~K}$ (Fig. 3, 2nd panel). A similar behavior is shown for higher stellar masses. This is however more extreme, implying that the transition to the atomic regime occurs earlier with a higher characteristic temperature. For instance, in the case of a supermassive star with $10^{3} M_{\odot}$, the transition to the atomic regime already occurs at $300 \mathrm{AU}$ with a peak temperature of $7000 \mathrm{~K}$.

For our reference model S1, the evolution of the species abundances is given in Fig. 3 (3rd panel), confirming the transition from the molecular to the atomic regime near $10 \mathrm{AU}$ as well as the subsequent build-up of molecular hydrogen due to high densities of the gas. The molecular hydrogen therefore starts affecting the cooling again around densities of $10^{16} \mathrm{~cm}^{-3}$. The viscous heating is initially balanced by the molecular cooling between 30 and 1000 AU (Fig. 3, bottom panel). Between 30 and $3 \mathrm{AU}$, the main cooling channel is due to $\mathrm{H}_{2}$ collisional dissociation cooling. In the central $3 \mathrm{AU}$, the contributions of viscous heating, continuum cooling as well as chemical heating and cooling strongly balance each other, and molecular hydrogen line cooling starts playing a role due to the increasing $\mathrm{H}_{2}$ abundance. However, it is also visible, for instance, in the $\mathrm{S} 2$ case that the $\mathrm{H}_{2}$ abundance is collisionally dissociated, as the critical temperature for $\mathrm{H}_{2}$ dissociation decreases with density and the disk evolves into a denser regime. We expect similar effects to occur in the scenarios S3 and S4, where the calculation becomes numerically unstable at this point as a result of stronger viscous heating.

\section{Chemical evolution for an initially atomic gas}

Now, we consider a case in which the gas in the disk is initially atomic. In this case, we adopt an initial molecular hydrogen abundance of $10^{-6}$ and an initial temperature of $10^{4} \mathrm{~K}$. Because of the higher temperature, we also assume an increased ionization degree of $10^{-6}$. In the following, we present the chemical evolution for an initially atomic gas, both in the case of a generic disk model as well as for a self-regulated disk.

\subsection{Results for a generic disk model with and without central source}

For the case of a generic disk model, we consider scenarios with and without a central source, with source masses of $10-10^{4} M_{\odot}$. We adopt here an accretion rate of $10^{-1} M_{\odot} \mathrm{yr}^{-1}$ to explore a case in which viscous heating is particularly relevant. We start with a characteristic surface density of $10 \mathrm{~g} \mathrm{~cm}^{-2}$ at $1000 \mathrm{AU}$. These disks are not necessarily self-gravitating, in particular, in the presence of a central source, and the viscosity to build up the disk thus needs to be provided by turbulence or (magneto-) hyrodynamical instabilities. A summary of the explored models is listed in Table 5. 

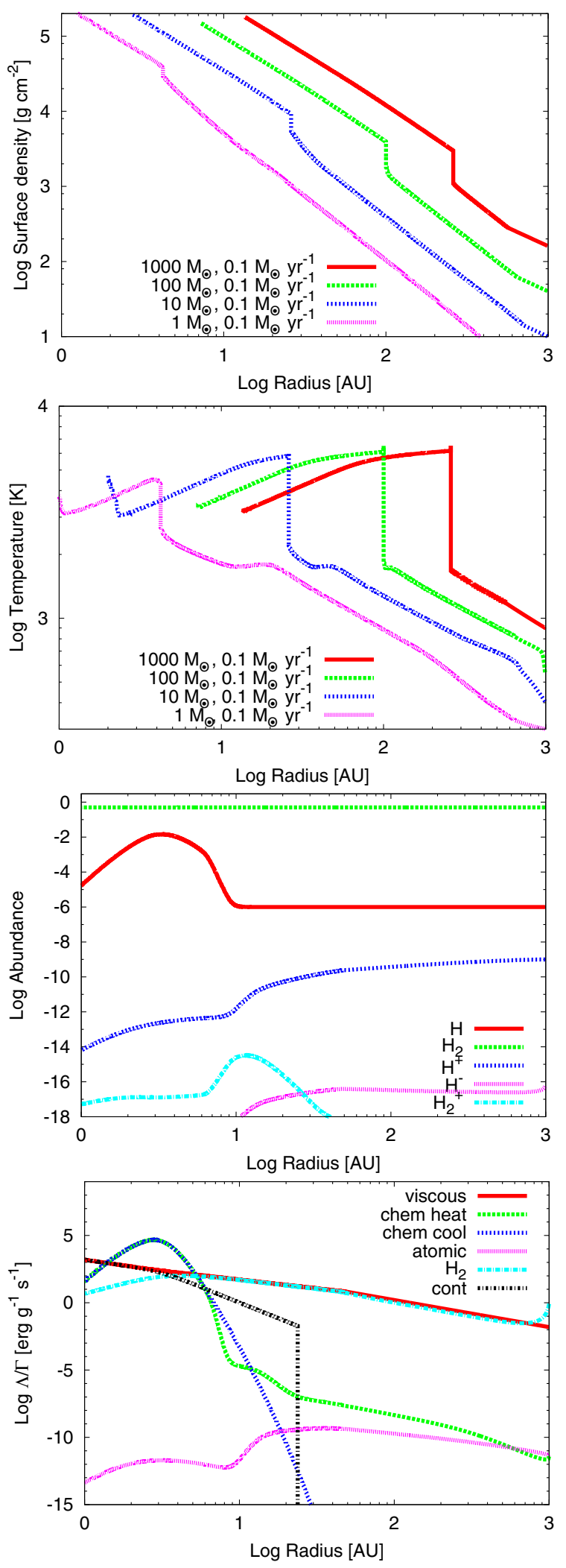

Fig. 3. Results for the self-regulated disk models with an initially molecular gas (Table 4), plus species abundances and heating/cooling rates for the reference case $S 1$ (self-regulated disk with central source of $1 M_{\odot}$ and an accretion rate of $\left.10^{-1} M_{\odot} \mathrm{yr}^{-1}\right)$. The top panel shows gas surface density as a function of radius. We refer to the caption of Fig. 2 for the description of the other panels.

The evolution of the gas temperature as a function of radius is shown in Fig. 4 (top panel). The temperature remains
Table 5. Models for a generic disk model with an initially atomic gas.

\begin{tabular}{lcccc}
\hline \hline Model & $M_{*}\left[M_{\odot}\right]$ & $\dot{M}\left[M_{\odot} \mathrm{yr}^{-1}\right]$ & $\Sigma\left[\mathrm{g} \mathrm{cm}^{-2}\right]$ & $T[\mathrm{~K}]$ \\
\hline H0 & 0 & $10^{-1}$ & 10 & $10^{4}$ \\
H1 & 10 & $10^{-1}$ & 10 & $10^{4}$ \\
H2 & 100 & $10^{-1}$ & 10 & $10^{4}$ \\
H3 & $10^{4}$ & $10^{-1}$ & 10 & $10^{4}$ \\
\hline
\end{tabular}

Table 6. Models for a self-regulated disk with initially atomic gas.

\begin{tabular}{lccc}
\hline \hline Model & $M_{*}\left[M_{\odot}\right]$ & $\dot{M}\left[M_{\odot} \mathrm{yr}^{-1}\right]$ & $\Sigma\left[\mathrm{g} \mathrm{cm}^{-2}\right]$ \\
\hline HS0 & 1 & $10^{-1}$ & 10 \\
HS1 & 10 & $10^{-1}$ & 10 \\
HS2 & 100 & $10^{-1}$ & 10 \\
HS3 & $10^{3}$ & $10^{-1}$ & 10 \\
\hline
\end{tabular}

high throughout the entire evolution, from $R=1000 \mathrm{AU}$ to $R=0.1 \mathrm{AU}$, but decreases slightly from 8000-9000 K initially to $6000-9000 \mathrm{~K}$ in the interior. The temperature increases slightly with central mass as a result of the enhanced viscous heating. The role of viscous heating is overall less pronounced compared to the initially molecular regime, as also found by Ferrara et al. (2013). In fact, the temperature remains high even in the absence of a central source, though may somewhat increase in the presence of viscous heating.

For a characteristic case with a central source of $10 M_{\odot}$ and an accretion rate of $10^{-1} M_{\odot} \mathrm{yr}^{-1}$ (model H1), the resulting species abundances are plotted in Fig. 4 (middle panel). The gas is atomic in the entire regime, and $\mathrm{H}_{2}$ remains strongly suppressed via collisional dissociation due to the high temperature. Within the central $10 \mathrm{AU}$, the $\mathrm{H}_{2}$ abundance starts increasing because of a mild decrease of the temperature, however, leading only to an abundance of $10^{-4}$ at $0.1 \mathrm{AU}$, which is insufficient to drive the cooling. The ionization degree only evolves very gradually as a function of radius, and is decreasing slightly due to the increasing densities. The viscous heating is balanced by the atomic cooling through almost the entire evolution (Fig. 4, bottom panel). In addition, chemical heating via three-body $\mathrm{H}_{2}$ formation becomes important only in the very central $0.3 \mathrm{AU}$, but is still balanced by the atomic line cooling. The molecular hydrogen cooling remains negligible throughout the entire evolution.

\subsection{Results for self-regulated disks}

Now, we finally explore the chemical evolution of initially atomic disks in a self-regulated scenario with $Q=1$. In this regime, we focus again on the case with a central source, as otherwise the evolution would be very similar to the case of a generic disk model. We consider here central sources with masses of $1-10^{3} M_{\odot}$, and characteristic accretion rates of $10^{-1} M_{\odot} \mathrm{yr}^{-1}$. We start with an initial surface density of $10 \mathrm{~g} \mathrm{~cm}^{-2}$, which adjusts early in the evolution because of the requirement of $Q=1$. As a result of the generally higher surface and number densities in this scenario, we restrict the evolution here to a minimum radius of $1 \mathrm{AU}$. In fact, considering stellar evolution models for supermassive stars, their typical extent may even exceed such a scale (Hosokawa et al. 2013). The overall models considered here are summarized in Table 6.

The surface density of the gas as a function of radius is given in Fig. 5 (top panel), showing that the surface density profile clearly steepens compared to a generic disk model, requiring a scaling as $\Sigma \propto R^{-1.5}$. Unlike the case with initially molecular 
D. R. G. Schleicher et al.: The chemical evolution of self-gravitating primordial disks
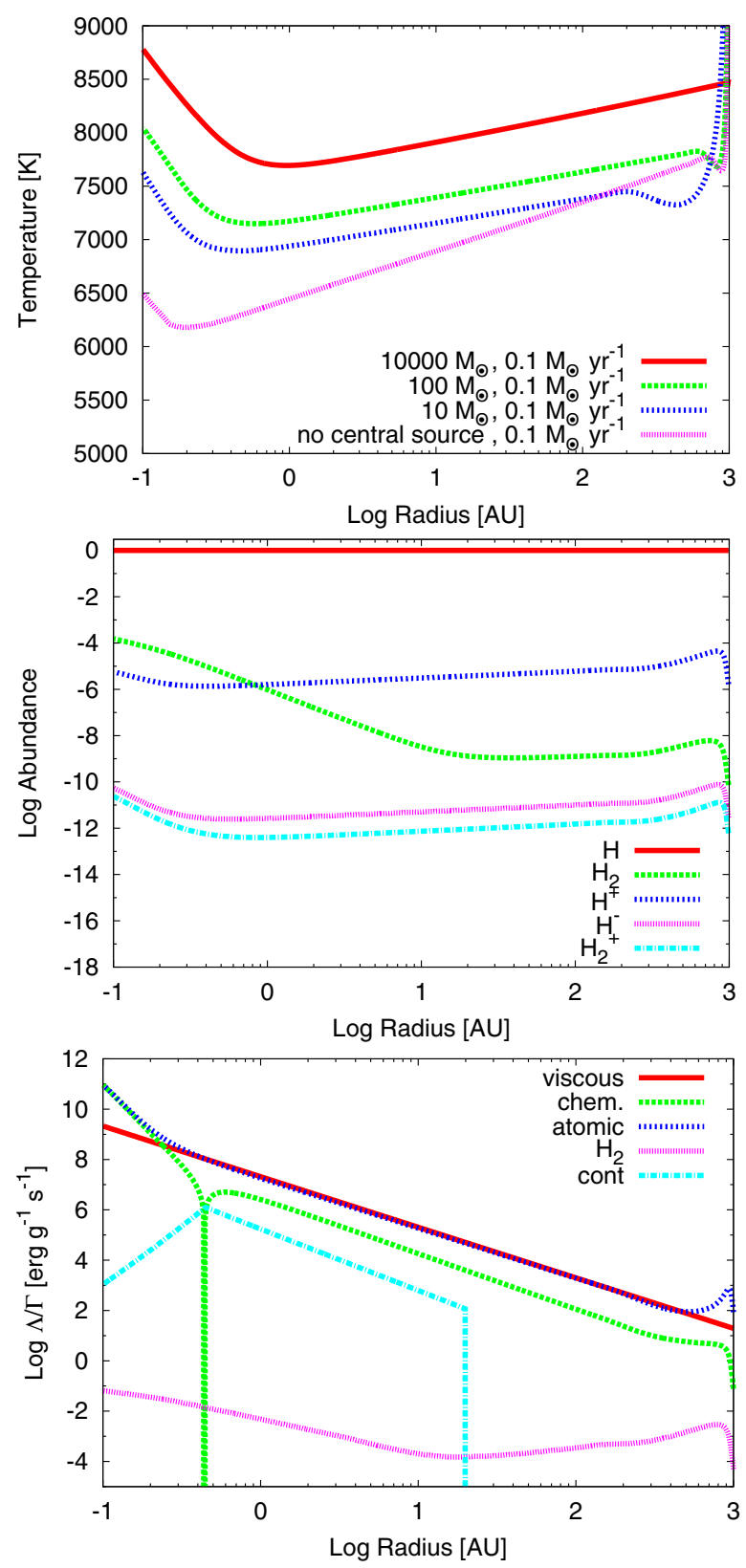

Fig. 4. Results for the generic disk model with an initially atomic gas (Table 5). The species abundances and heating/cooling rates are given for the $\mathrm{H} 1$ (generic disk model with central source of $10 M_{\odot}$ and an accretion rate of $\left.10^{-1} M_{\odot} \mathrm{yr}^{-1}\right)$. We refer to the caption of Fig. 1 for the description of the panels.

cooling, the profile strongly resembles a power law with no particular features, as the temperature evolution is generally more gradual and mostly without strong transitions. The temperature initially remains high, while some continuum cooling may set in between 60 and $10 \mathrm{AU}$ (Fig. 5, 2nd panel). The temperature then decreases more steeply toward $\sim 3000 \mathrm{~K}$. For central sources with 1 and $10 M_{\odot}$, we find that the temperature subsequently increases again, as a result of viscous heating and $\mathrm{H}_{2}$ collisional dissociation. For higher central masses, the calculation becomes unstable at the point of this transition due to the correspondingly higher heating and cooling rates. In principle, we expect a similar evolution.

The chemical evolution is given for a characteristic case with a central source of $1 M_{\odot}$ and an accretion rate of $10^{-1} M_{\odot} \mathrm{yr}^{-1}$, corresponding to model HSO (Fig. 5, 3rd panel). While the gas
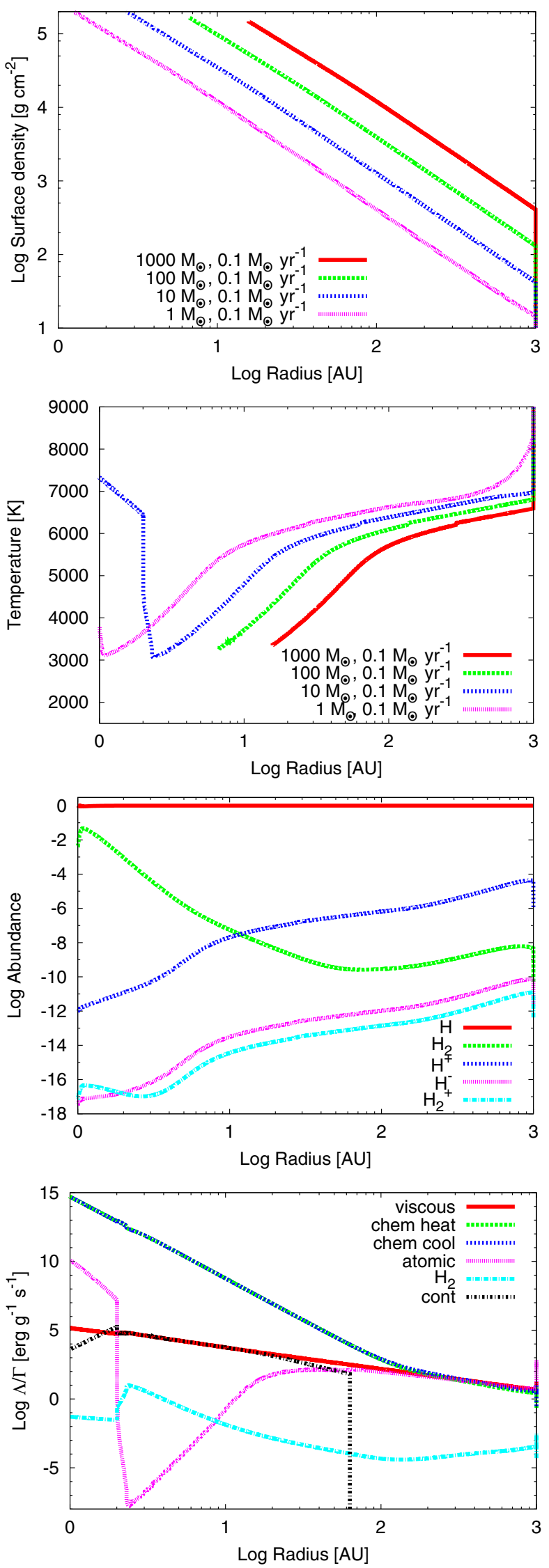

Fig. 5. Results for the self-regulated disk models with an initially atomic gas (Table 6). The species abundances and heating/cooling rates are given for the HS0 (self-regulated disk with central source of $1 M_{\odot}$ and an accretion rate of $\left.10^{-1} M_{\odot} \mathrm{yr}^{-1}\right)$. We refer to the caption of Fig. 3 for the description of the panels. 
is atomic in the entire range, the $\mathrm{H}_{2}$ abundance increases significantly from $\sim 10^{-9}$ at $\sim 50 \mathrm{AU}$, reaching a peak value close to 0.1 at $1 \mathrm{AU}$. The ionization degree initially decreases only slightly with density between 1000 and $10 \mathrm{AU}$, and then drops more significantly toward $10^{-12}$ because of lower temperatures.

As shown in Fig. 5 (bottom panel), the viscous heating is balanced by atomic hydrogen cooling between 1000 and $30 \mathrm{AU}$. In the interior, the contribution of the continuum cooling is subsequently close to the viscous heating. In this regime, the chemical heating and cooling as well as the contributions of viscous heating and continuum cooling strongly balance each other, and the temperature decrease is driven by molecular hydrogen cooling. The characteristic temperature for $\mathrm{H}_{2}$ dissociation, however, depends on the density, and the critical point for $\mathrm{H}_{2}$ dissociation is indeed reached at $R \sim 1 \mathrm{AU}$, leading to the dissociation of molecular hydrogen and a resulting increase of the temperature.

Also, in this case, the role of viscous heating is therefore less pronounced for the initially atomic than for the initially molecular regime. However, as a result of the higher gas densities in the self-gravitating regime, the $\mathrm{H}_{2}$ formation is enhanced for these cases so that the cooling is initially somewhat enhanced. The viscous heating prevents the gas from becoming molecular again in the central 1-10 AU, therefore, somewhat contributing to the stability in the very central region. This behavior concerns relatively small scales. A supermassive star with $\sim 10^{4} M_{\odot}$ may already have radii up to $\sim 100 \mathrm{AU}$ (Hosokawa et al. 2013), providing a natural cutoff for the inner disk. It thus appears likely that the viscous heating only has a minor effect in the initially atomic regime.

\section{Summary and discussion}

We have developed a one-zone framework to describe the chemical and thermal evolution of primordial disks, depending on the mechanism that provides the viscosity of the disk. Assuming a sufficient viscosity is always available as a result of turbulence and/or magnetic fields (see, e.g., Balbus \& Papaloizou 1999; Hawley 2000), we derived the generic disk model under the assumption of stationarity and axisymmetry as well as a surface density profile $\Sigma \propto R^{-1}$, corresponding to a Mestel disk. In this framework, the effective viscosity of the disk follows from the requirement of maintaining the accretion rate and transport of angular momentum, therefore, providing the amount of viscous heating (see, e.g., Lodato 2007; Inayoshi \& Haiman 2014; Latif $\&$ Schleicher 2015a). We further distinguish between scenarios in which the rotation profile is determined by the self-gravity of the disk and scenarios in which gravity is dominated by the central source.

If rotation is dominated by the central source, it follows that the disk becomes gravitationally stable on smaller scales because of the relation $\Sigma \propto R^{-1}$. In particular, in the regime of high accretion rates, however, one may expect that self-gravity contributes signficantly to the transport of angular momentum (e.g., Begelman \& Shlosman 2009). Hence, we also consider a self-regulated disk model, in which the disk viscosity is due to gravitational instabilities, and, therefore, requires a scenario with Toomre $Q \sim 1$. In these cases, we obtain a steeper profile for the surface density of the disk in a marginally stable state. We refer here to Lodato (2007) for a review on the properties of such disks.

The resulting evolution has been explored for a range of stellar masses and accretion rates, considering both conventional Pop. III star formation (e.g., Abel et al. 2002; Bromm \& Loeb 2003; Yoshida et al. 2008) as well as the formation of supermassive primordial stars (e.g., Lodato \& Natarajan 2006; Regan \& Haehnelt 2009; Latif et al. 2013a; Ferrara et al. 2014). As chemical initial conditions, we explored both an initially molecular gas, as expected for instance in minihalos (e.g., Abel et al. 2002; Bromm \& Loeb 2003; Yoshida et al. 2008), and an initially atomic gas. In more massive halos, the chemical evolution depends on the ambient radiation background and is typically molecular for moderate values and atomic for very strong values of the radiation background (e.g., Omukai 2001; Greif et al. 2008; Shang et al. 2010; Schleicher et al. 2010; Latif et al. 2011, 2015a).

In case of the initially molecular gas, we find that viscous heating plays a crucial role in the presence of a central source and accretion rates of $\sim 10^{-1} M_{\odot} \mathrm{yr}^{-1}$, both for the generic and self-regulated disk model. For a central star with about $10 M_{\odot}$, the viscous heating leads to the collisional dissociation of molecular hydrogen on scales of $\sim 10 \mathrm{AU}$. The effect is further enhanced for supermassive stars with $\sim 10^{4} M_{\odot}$, where the molecular gas is already dissociated on scales of $\sim 1000 \mathrm{AU}$, which strongly contributes to the stabilization of the central region. Even if fragmentation occurs, the resulting clumps are more massive, and are expected to rapidly migrate toward the central region (Inayoshi \& Haiman 2014; Latif \& Schleicher 2015b). The transition toward an atomic gas also increases the opacity, which is typically on the order of $1 \mathrm{~cm}^{2} \mathrm{~g}^{-1}$ at a temperature of $10^{4} \mathrm{~K}$. Such a transition can have a relevant impact on the structure of the disk and increase the overall stability. However, fragmentation may be more efficient on larger scales where the gas is molecular and the migration time is also increasing with the distance from the central object. It is thus certainly viable that fragmentation occurs on some scale, which could potentially contribute to the formation of a starburst ring around the central object (Lodato \& Natarajan 2006). In such a case, the further accretion may be regulated by the interplay of gravitational instabilities with stellar feedback (Kawakatu \& Wada 2009; Wutschik et al. 2013).

We further explored the case in which the gas is initially atomic. In case of the generic disk model, it then remains atomic, as collisional dissociation of molecular hydrogen is efficient at the temperatures of atomic hydrogen cooling (Inayoshi \& Omukai 2012). Because of the high temperatures, the overall cooling rates are considerably enhanced and the effect of viscous heating is therefore less relevant in this regime, as also described by Ferrara et al. (2013) for a hot metal-enriched gas. In the case of a self-gravitating disk model, we find that some $\mathrm{H}_{2}$ may build up again toward the central region as a result of the higher densities obtained in this regime. However, the characteristic temperatures are still above $\sim 3000 \mathrm{~K}$ throughout the evolution. As the characteristic temperature for $\mathrm{H}_{2}$ collision dissociation decreases with increasing density, we find that the $\mathrm{H}_{2}$ dissociates again after an initial buildup. Overall, the gas essentially remains atomic for most of the evolution.

The potential impact of viscous heating is well known, for instance, in the case of quasar accretion disks, where the resulting heat input leads to the presence of a hot gas over a large range of scales (e.g., Goodman 2003; Goodman \& Tan 2004). In the context of primordial star formation, these kinds of effects have not been strongly considered. The one-zone models exploring the chemical evolution have typically assumed a free-fall collapse (e.g., Omukai 2001; Omukai et al. 2005; Glover \& Abel 2008; Glover 2015), while simulations were usually restricted to the early stages of disk formation. For instance, Clark et al. (2011) were following the evolution until $\sim 1 M_{\odot}$ of mass had turned into stars, while Greif et al. (2011) stopped the evolution when 
a $\sim 10 M_{\odot}$ star was formed, and still had a moderate accretion rate of $10^{-2} M_{\odot}$. In a follow-up investigation at higher resolution, they evolved the simulation to stellar masses of $\sim 1 M_{\odot}$, and found that heating had occurred due to both the accretion onto the protostar and shocks close to the spiral arm (Greif et al. 2012). It is conceivable that these are the first signatures of viscous heating, even though they are still at an early stage. In the context of supermassive stars, we emphasize that the majority of runs already started from an atomic gas, which typically remained atomic during the further evolution (e.g., Regan \& Haehnelt 2009; Latif et al. 2013a,c; Prieto et al. 2013; Regan et al. 2014; Becerra et al. 2015). The longer-term evolution in the presence of molecular cooling has been explored in simulations by Latif et al. (2014b) and Latif \& Volonteri (2015). These simulations show that high accretion rates can be maintained, even though they do not resolve the scales considered here.

The results obtained here are particularly important for the formation of a massive central object, as the enhanced temperature in the atomic gas increases the stability in the interior of the disk and helps to maintain a high accretion rate. The high accretion rate also alleviates the need for a strong external radiation field, typically expressed through a critical value $J_{\text {crit }}$, as a means of providing an atomic gas. We expect these results to be particularly relevant in the case of massive central objects of at least $10 M_{\odot}$ and high accretion rates of $\sim 10^{-1} M_{\odot} \mathrm{yr}^{-1}$. The nature of the phenomenon discussed here and, in particular, the characteristic scaling of the viscous heating rate as $R^{-3}$ leads us to the expectation that such a transition should generally occur. This is the case even though it may be shifted, for instance, if the surface density is enhanced and thus also affects the ability of the gas to cool. Even in the case of fragmentation and a more turbulent flow, we expect gas velocities of approximately the Keplerian velocity to occur, leading to the formation of strong shocks and a transition toward the atomic cooling regime.

Of course, the models here are still based on simplifying assumptions, such as the stationarity and axisymmetry of the disk, which can only hold at an approximate level. It is therefore important to investigate the evolution in these disks in 3D simulations, following the interplay of chemistry, heating, and cooling along with the gravitational dynamics to assess the impact on fragmentation. For these time-dependent models, the viscosity of the disk can no longer be obtained from the assumption of stationarity, but one may have to adopt explicit parametrizations of the viscosity in self-gravitating disks as derived, for instance, by Rafikov (2015). As a result of the timescales involved in the evolution of such disks, we expect that such studies cannot be pursued in cosmological simulations, but that different setups are needed to investigate the stability of self-gravitating primordial disks with an already a massive protostar. Depending on the mass and accretion rate of the protostar, radiation feedback may also become relevant even though it may predominantly affect the regions outside the disk (e.g., Hosokawa et al. 2011; Hirano et al. 2014). In the case of rapid accretion, it was found that the UV feedback from protostars is likely negligible, as they are expected to have cool atmospheres like a red giant (Hosokawa et al. 2013; Schleicher et al. 2013). Previous investigations have further shown that $\alpha$ disk models with an optically thin cooling are thermally unstable (Cannizzo \& Wheeler 1984; Kato et al. 1998). In fact, the models pursued here are more complex and, in particular, do not employ a constant $\alpha$, as the disk viscosity is derived from Eq. (9). It is nevertheless conceivable that thermal instabilities may play a relevant role in their evolution, which needs to be explored in further detail.
Beyond the primordial case, the influence of dust cooling is potentially significant at high densities (e.g., Schneider et al. 2003, 2006, 2012; Omukai et al. 2005, 2008; Cazaux \& Spaans 2009; Dopcke et al. 2011, 2013). Most of these investigations have employed one-zone models assuming a free-fall collapse, while we have shown here that the heating mechanism is considerably different in a rotating disk around a central protostar. In this regime, the role of dust cooling requires further investigations to understand the fragmentation behavior at later stages of the evolution. The latter can be important both for the formation of the first low-mass stars (e.g., Schneider et al. 2012; Klessen et al. 2012) and for the formation scenarios of massive black holes (Omukai et al. 2008; Ferrara et al. 2013). Even if fragmentation occurs, a black hole may possibly form, as long as the cluster is fed via substantial inflows and gravitational instabilities occur (Alexander \& Natarajan 2014; Latif et al. 2015b).

Acknowledgements. D.R.G.S. and S.B. are thankul for funding through the DFG priority program "The Physics of the Interstellar Medium" (projects SCHL 1964/1-1, SCHL 1964/1-2 and BO 4113/1-2). M.L. received funding from the "BLACK" project funded via the European Research Council under the European Community's Seventh Framework Programme (FP7/2007-2013 Grant Agreement No. 614199, project "BLACK"). T.G. acknowledges the Centre for Star and Planet Formation funded by the Danish National Research Foundation.

\section{References}

Abel, T., Bryan, G. L., \& Norman, M. L. 2002, Science, 295, 93

Agarwal, B., Dalla Vecchia, C., Johnson, J. L., Khochfar, S., \& Paardekooper, J.-P. 2014, MNRAS, 443, 648

Agarwal, B., Smith, B., Glover, S., Natarajan, P., \& Khochfar, S. 2015, MNRAS, submitted [arXiv: 1504.04042$]$

Alexander, T., \& Natarajan, P. 2014, Science, 345, 1330

Bañados, E., Venemans, B. P., Morganson, E., et al. 2014, AJ, 148, 14

Balbus, S. A., \& Papaloizou, J. C. B. 1999, ApJ, 521, 650

Becerra, F., Greif, T. H., Springel, V., \& Hernquist, L. E. 2015, MNRAS, 446, 2380

Beckwith, S. V. W., Sargent, A. I., Chini, R. S., \& Guesten, R. 1990, AJ, 99, 924

Begelman, M. C., \& Shlosman, I. 2009, ApJ, 702, L5

Bertin, G. 1997, ApJ, 478, L71

Bovino, S., Grassi, T., Latif, M. A., \& Schleicher, D. R. G. 2013, MNRAS, 434, L36

Bovino, S., Grassi, T., Schleicher, D. R. G., \& Latif, M. A. 2014a, ApJ, 790, L35 Bovino, S., Latif, M. A., Grassi, T., \& Schleicher, D. R. G. 2014b, MNRAS, 441, 2181

Bovino, S., Schleicher, D. R. G., \& Grassi, T. 2014c, A\&A, 561, A13

Bromm, V., \& Loeb, A. 2003, ApJ, 596, 34

Cannizzo, J. K., \& Wheeler, J. C. 1984, ApJS, 55, 367

Cazaux, S., \& Spaans, M. 2009, A\&A, 496, 365

Cen, R. 1992, ApJS, 78, 341

Clark, P. C., Glover, S. C. O., \& Klessen, R. S. 2008, ApJ, 672, 757

Clark, P. C., Glover, S. C. O., Smith, R. J., et al. 2011, Science, 331, 1040

Dijkstra, M., Haiman, Z., Mesinger, A., \& Wyithe, J. S. B. 2008, MNRAS, 391, 1961

Dijkstra, M., Ferrara, A., \& Mesinger, A. 2014, MNRAS, 442, 2036

Dopcke, G., Glover, S. C. O., Clark, P. C., \& Klessen, R. S. 2011, ApJ, 729, L3 Dopcke, G., Glover, S. C. O., Clark, P. C., \& Klessen, R. S. 2013, ApJ, 766, 103 Fan, X., Narayanan, V. K., Lupton, R. H., et al. 2001, AJ, 122, 2833

Fan, X., Hennawi, J. F., Richards, G. T., et al. 2004, AJ, 128, 515

Fan, X., Strauss, M. A., Richards, G. T., et al. 2006, AJ, 131, 1203

Ferrara, A., Haardt, F., \& Salvaterra, R. 2013, MNRAS, 434, 2600

Ferrara, A., Salvadori, S., Yue, B., \& Schleicher, D. 2014, MNRAS, 443, 2410 Forrey, R. C. 2013, ApJ, 773, L25

Fromang, S., Balbus, S. A., Terquem, C., \& De Villiers, J.-P. 2004, ApJ, 616, 364

Glover, S. C. O. 2015, MNRAS, 453, 2901

Glover, S. C. O., \& Abel, T. 2008, MNRAS, 388, 1627

Goodman, J. 2003, MNRAS, 339, 937

Goodman, J., \& Tan, J. C. 2004, ApJ, 608, 108

Grassi, T., Bovino, S., Schleicher, D. R. G., et al. 2014, MNRAS, 439, 2386

Greif, T. H. 2015, Comput. Astrophys. Cosmol., 2, 3

Greif, T. H., Johnson, J. L., Klessen, R. S., \& Bromm, V. 2008, MNRAS, 387, 1021 
Greif, T. H., Springel, V., White, S. D. M., et al. 2011, ApJ, 737, 75

Greif, T. H., Bromm, V., Clark, P. C., et al. 2012, MNRAS, 424, 399

Hawley, J. F. 2000, ApJ, 528, 462

Hirano, S., Hosokawa, T., Yoshida, N., et al. 2014, ApJ, 781, 60

Hosokawa, T., Omukai, K., Yoshida, N., \& Yorke, H. W. 2011, Science, 334, 1250

Hosokawa, T., Yorke, H. W., Inayoshi, K., Omukai, K., \& Yoshida, N. 2013, ApJ, 778, 178

Inayoshi, K., \& Haiman, Z. 2014, MNRAS, 445, 1549

Inayoshi, K., \& Omukai, K. 2012, MNRAS, 422, 2539

Kato, S., Fukue, J., \& Mineshige, S. 1998, Black-hole accretion disks (Kyoto University Press)

Kawakatu, N., \& Wada, K. 2009, ApJ, 706, 676

Klessen, R. S., Glover, S. C. O., \& Clark, P. C. 2012, MNRAS, 421, 3217

Koushiappas, S. M., Bullock, J. S., \& Dekel, A. 2004, MNRAS, 354, 292

Latif, M. A., \& Schleicher, D. R. G. 2015a, MNRAS, 449, 77

Latif, M. A., \& Schleicher, D. R. G. 2015b, A\&A, 578, A118

Latif, M. A., \& Volonteri, M. 2015, MNRAS, 452, 1026

Latif, M. A., Schleicher, D. R. G., Spaans, M., \& Zaroubi, S. 2011, A\&A, 532, A66

Latif, M. A., Schleicher, D. R. G., Schmidt, W., \& Niemeyer, J. 2013a, MNRAS, 433,1607

Latif, M. A., Schleicher, D. R. G., Schmidt, W., \& Niemeyer, J. 2013b, ApJ, 772, L3

Latif, M. A., Schleicher, D. R. G., Schmidt, W., \& Niemeyer, J. C. 2013c, MNRAS, 436, 2989

Latif, M. A., Bovino, S., Van Borm, C., et al. 2014a, MNRAS, 443, 1979

Latif, M. A., Schleicher, D. R. G., Bovino, S., Grassi, T., \& Spaans, M. 2014b, ApJ, 792, 78

Latif, M. A., Bovino, S., Grassi, T., Schleicher, D. R. G., \& Spaans, M. 2015a, MNRAS, 446, 3163

Latif, M. A., Omukai, K., Habouzit, M., Schleicher, D. R. G., \& Volonteri, M. 2015b, MNRAS, submitted [arXiv: 1509.07034]

Lenzuni, P., Chernoff, D. F., \& Salpeter, E. E. 1991, ApJS, 76, 759

Lodato, G. 2007, Nuovo Cimento Rivista Serie, 30, 293

Lodato, G., \& Natarajan, P. 2006, MNRAS, 371, 1813

Mayer, L., Kazantzidis, S., Escala, A., \& Callegari, S. 2010, Nature, 466, 1082
Mayer, L., Fiacconi, D., Bonoli, S., et al. 2015, ApJ, 810, 51

Mortlock, D. J., Warren, S. J., Venemans, B. P., et al. 2011, Nature, 474, 616

Natarajan, P. 2011, The formation and evolution of massive black hole seeds in the early Universe, eds. D. J. Saikia, \& V. Trimble (World Scientific Publishing Co), 191

Omukai, K. 2000, ApJ, 534, 809

Omukai, K. 2001, ApJ, 546, 635

Omukai, K., Tsuribe, T., Schneider, R., \& Ferrara, A. 2005, ApJ, 626, 627

Omukai, K., Schneider, R., \& Haiman, Z. 2008, ApJ, 686, 801

Peters, T., Schleicher, D. R. G., Smith, R. J., Schmidt, W., \& Klessen, R. S. 2014, MNRAS, 442, 3112

Prieto, J., Jimenez, R., \& Haiman, Z. 2013, MNRAS, 436, 2301

Rafikov, R. R. 2015, ApJ, 804, 62

Regan, J. A., \& Haehnelt, M. G. 2009, MNRAS, 396, 343

Regan, J. A., Johansson, P. H., \& Haehnelt, M. G. 2014, MNRAS, 439, 1160

Safranek-Shrader, C., Milosavljević, M., \& Bromm, V. 2014, MNRAS, 438, 1669

Safranek-Shrader, C., Montgomery, M., Milosavljevic, M., \& Bromm, V. 2015, MNRAS, 455, 3288

Schleicher, D. R. G., Spaans, M., \& Glover, S. C. O. 2010, ApJ, 712, L69

Schleicher, D. R. G., Palla, F., Ferrara, A., Galli, D., \& Latif, M. 2013, A\&A, 558, A59

Schneider, R., Ferrara, A., Salvaterra, R., Omukai, K., \& Bromm, V. 2003, Nature, 422,869

Schneider, R., Salvaterra, R., Ferrara, A.. \& Ciardi, B. 2006, MNRAS, 369, 825

Schneider, R., Omukai, K., Limongi, M., et al. 2012, MNRAS, 423, L60

Sethi, S., Haiman, Z., \& Pandey, K. 2010, ApJ, 721, 615

Shang, C., Bryan, G. L., \& Haiman, Z. 2010, MNRAS, 402, 1249

Sugimura, K., Omukai, K., \& Inoue, A. K. 2014, MNRAS, 445, 544

Susa, H., Hasegawa, K., \& Tominaga, N. 2014, ApJ, 792, 32

Tanaka, K. E. I., \& Omukai, K. 2014, MNRAS, 439, 1884

Toomre, A. 1964, ApJ, 139, 1217

Turk, M. J., Abel, T., \& O'Shea, B. 2009, Science, 325, 601

Van Borm, C., \& Spaans, M. 2013, A\&A, 553, L9

Volonteri, M., \& Bellovary, J. 2012, Rep. Prog. Phys., 75, 124901

Wutschik, S., Schleicher, D. R. G., \& Palmer, T. S. 2013, A\&A, 560, A34

Yoshida, N., Omukai, K., \& Hernquist, L. 2008, Science, 321, 669 